\title{
ON H. SIMPSON'S SIX-CONIC THEOREM
}

\author{
R. H. F. DENNISTON
}

(received 29 August 1962)

H. Simpson [1] has proved a theorem about six points $P_{1}, \ldots, P_{B}$ (no three in line, not all on a conic) of the real projective plane. He calls $P_{1}$ an "in-point" or an "out-point", according as it lies inside or outside the conic (denoted by a Roman figure I) through the other five points; and so on. The theorem is that there are either two, three, or six in-points.

Other properties of the six points may be studied by the same method. Let us describe the line $P_{1} P_{2}$ as "touched" when there are two real conics passing through $P_{3}, P_{4}, P_{5}, P_{6}$ and touching the line. It suffices to look at the pairs of points in which the line is met by two pairs of opposite sides of the quadrangle: the line is touched if and only if these two pairs do not separate each other.

Through $P_{1}$ there are five pairs of lines which are known to belong to an involution pencil: namely, $P_{1} P_{2}$ and the tangent to the conic II, and so on. Let us describe $P_{1}$ as "elliptic" or "hyperbolic", according to the type of this involution pencil; it again suffices to look at two of the pairs of lines, and see whether they separate each other or not.

Suppose that $P_{2}, \ldots, P_{6}$ are fixed (without loss of generality, as in [1], they may be arranged in order round a circle), and that $P_{1}$ moves about. As $P_{1}$ crosses $P_{2} P_{3}$, we know from [1] that each of $P_{4}, P_{5}, P_{6}$ (but no other point) changes from in-point to out-point, or vice versa. We can also see that, as $P_{1}$ crosses $P_{2} P_{3}$, each of the lines $P_{1} P_{2}, P_{1} P_{3}, P_{2} P_{3}, P_{4} P_{5}$, $P_{4} P_{6}, P_{5} P_{6}$ (but none of the other nine joins) changes from touched to untouched, or vice versa; and that each of $P_{1}, P_{2}, P_{3}$ (but no other point) changes from elliptic to hyperbolic, or vice versa. As $P_{1}$ crosses the conic I, each of the six points changes from in-point to out-point (or vice versa), and also from elliptic to hyperbolic (or vice versa): but the touched and untouched lines remain as they are. Using these considerations, we can study the various cases that arise when $P_{1}$ lies in different regions of the plane. Without much labour, we can verify the following conclusions:

When there are two in-points, any join of two in-points or of two out-points is touched: any join of an in-point and an out-point is untouched.

When there are three in-points, the contrary is true; the only touched lines are those joining an in-point and an out-point. 
When there are six in-points, all the lines are touched.

When there are two in-points, they are elliptic and the out-points hyperbolic. When there are three in-points, they are hyperbolic and the out-points elliptic. When the points are all in-points, they are all elliptic.

These facts might have been easily deduced from a theorem [2] about a cubic surface: but it is not without interest that Simpson's method suffices to prove them.

\section{- Appendix}

The referee has asked for the theorem about the involution pencil at $P_{1}$ to be substantiated. I cannot find any reference to it, and quite possibly it has never been published: so I give a proof.

Let $P_{1} T_{2}, P_{1} T_{3}, \ldots, P_{1} T_{6}$ be the tangents at $P_{1}$ to the conics II, III, IV, V, VI: it is required to prove that the pairs $\left(P_{1} P_{2}, P_{1} T_{2}\right), \ldots$, $\left(P_{1} P_{B}, P_{1} T_{6}\right)$ belong to an involution pencil. But, since the pairs are defined symmetrically, and two pairs determine an involution, it suffices to prove that three of the pairs belong to an involution pencil. Consider the pairs $\left(P_{1} P_{2}, P_{1} T_{2}\right),\left(P_{1} P_{3}, P_{1} T_{3}\right),\left(P_{1} P_{4}, P_{1} T_{4}\right)$; and, since the theorem is projective and is independent of reality, let us take $P_{6}$ and $P_{6}$ to be the circular points.

We then have II, III, IV as the circles $P_{1} P_{3} P_{4}, P_{1} P_{2} P_{4}, P_{1} P_{2} P_{3}$ (with diameters $d_{2}, d_{3}, d_{4}$, say), and $P_{1} T_{2}, P_{1} T_{3}, P_{1} T_{4}$ as the tangents at $P_{1}$; and so we have six formulae of the form

And then

$$
P_{1} P_{3}=d_{2} \sin T_{2} P_{1} P_{3} \text {. }
$$

$$
\begin{aligned}
P_{1}\left\{T_{2} P_{3} T_{3} P_{4}\right\} & =\frac{\sin T_{2} P_{1} P_{3} \sin T_{3} P_{1} P_{4}}{\sin T_{2} P_{1} P_{4} \sin T_{3} P_{1} P_{3}} \\
& =\frac{P_{1} P_{3}}{d_{3} \sin T_{3} P_{1} P_{8}} \\
& =P_{1}\left\{P_{2} T_{3} P_{3} T_{4}\right\},
\end{aligned}
$$

and the three pairs belong to an involution pencil.

\section{References}

[1] H. Simpson, On F. Supnick's Six-Conic Theorem, Proc. Amer. Math. Soc., Vol. 12 (1861), p. 931.

[2] B. Segre, The Non-Singular Cubic Surfaces, Oxford, Clarendon Press (1942), p. 48, subsection (i).

The University College, Ibadan

and the University of Canterbury (N.Z.) 